\title{
Semi-analytical methods of solution for the BGK-Boltzmann equation describing sound wave propagation in binary gas mixtures
}

\author{
Silvia Lorenzani
}

\begin{abstract}
In the present paper, the propagation of high-frequency sound waves in binary gas mixtures is investigated by using the linearized Boltzmann equation based on a Bhatnagar-Gross-Krook (BGK)-type approach and diffuse reflection boundary conditions. It is shown that the semi-analytical methods of solution considered for the BGK-Boltzmann equations can be used to simplify the numerical procedures for solving our problem and gain insight into the qualitative behavior of the solution.
\end{abstract}

\section{Introduction}

In the low-frequency limit, the problem concerning the propagation of sound waves in gas mixtures is correctly described by the Navier-Stokes, Fourier, and Fick equations [16], [17]. If the excitation frequency $\omega$ of the sound wave becomes sufficiently high, the classical continuum approach fails even at ordinary densities, because $\omega^{-1}$ can become of the order of the molecular mean free time. Therefore, in the case of rarefied gas dynamics (or equivalently, in the case of a highly oscillatory phenomenon), there is no recourse but to turn to the kinetic theory and the Boltzmann equation [6], [25], [19], [10], [15]. In the sixties, Grad [12] conjectured that when a binary gas mixture is made up of species with very different molecular masses, under appropriate circumstances, the two components of such a mixture can support different temperatures, due to the slow exchange of kinetic energy between light and heavy species. Two-temperature continuum equations, necessary to replace those of hydrodynamics in part of the continuum regime, have been developed by a number of authors. From such "two-temperature hydrodynamics", Huck and Johnson [14] predicted the possibility of several simultaneous sound modes in a disparate-mass gas mixture (composed of very heavy plus very light molecules),

Silvia Lorenzani

Dipartimento di Matematica, Politecnico di Milano, Piazza Leonardo da Vinci 32, 20133 Milan, Italy, e-mail: silvia.lorenzani@polimi.it 
for a narrow range of compositions at high enough frequencies. These predictions were later confirmed experimentally by Bowler [3]. However, the identity of these interfering modes has never been completely understood over the years, partly due to the inadequacy of the investigation tools used.

The kinetic description of a mixture of gases with different particle masses (and possibly with different internal energies) is not a trivial generalization of the classical Boltzmann theory for a single gas, since the collision operators have to take into account exchanges of momentum and energy among the different species (and also mass exchanges, in the case of reacting mixtures). Therefore, since the Boltzmann equations are quite awkward to deal with, consistent simpler models have been proposed in the literature. Among them, the McCormack model [20] has been over the years the most widely used to study a great variety of problems, such as Couette and Poiseuille flows, thermal creep, and heat transfer for mixtures, since all transport coefficients (i.e., viscosity, thermal conductivity, diffusion, and thermal diffusion ratio) can be correctly obtained from it applying the Chapman-Enskog procedure [21], [22]. Moreover, McCormack derived a model for the cross-collision operator of a general multicomponent monatomic mixture applicable to different intermolecular force laws.

Instead, in the present paper, we have chosen to perform our analysis by using the BGK relaxation model proposed by Andries et al. [1] and generalized by Groppi and Spiga [13] even to nonconservative collisions, that has been proven to be well posed from the mathematical point of view (correct Boltzmann collision invariants and Maxwellian equilibria are properly recovered, and the $\mathrm{H}$ theorem is fulfilled). Even if the BGK-Boltzmann equation for mixtures generally cannot match all the transport coefficients simultaneously, for the specific problem at hand, we will prove that the BGK model is able to produce results in close agreement with those obtained with more refined kinetic models. The advantage is that the equations which describe the BGK model are much simpler than those which describe, for instance, the McCormack model, allowing also a semi-analytical representation of the solution.

\section{Linearized BGK Model}

Let us consider a binary gaseous mixture confined between two flat, infinite, and parallel plates located at $z^{\prime}=-d / 2$ and $z^{\prime}=d / 2$. Both boundaries are held at the same constant temperature. The upper wall of the channel (located at $z^{\prime}=d / 2$ ) is fixed while the lower one (located at $z^{\prime}=-d / 2$ ) harmonically oscillates in the $z^{\prime}$ direction (normal to the wall itself) with angular frequency $\omega^{\prime}$ (the corresponding period being $\left.T^{\prime}=2 \pi / \omega^{\prime}\right)$. The velocity $U_{w}^{\prime}$ of the oscillating plate depends on time $t^{\prime}$ through the formula

$$
U_{w}^{\prime}\left(t^{\prime}\right)=U_{0}^{\prime} \sin \left(\omega^{\prime} t^{\prime}\right),
$$


where it is assumed that the amplitude $U_{0}^{\prime}$ is very small compared to the characteristic molecular velocity of the mixture given by

$$
v_{0}=\sqrt{2 k T_{0} / m}
$$

with $k$ being the Boltzmann constant, $m$ being the mean molecular mass of the mixture and $T_{0}$ being the equilibrium temperature of the mixture. Under these conditions, the Boltzmann equation modeling the gaseous mixture motion inside the channel can be linearized by representing the distribution functions $f^{s}(s=1,2)$ of both species as follows:

$$
f^{s}=f_{0}^{s}\left(1+h^{s}\right), \quad\left|h^{s}\right|<<1,
$$

with $f_{0}^{s}$ being the Maxwellian configuration

$$
f_{0}^{s}=n_{0}^{s}\left(\frac{m^{s}}{2 \pi k T_{0}}\right)^{3 / 2} \exp \left[-\frac{m^{s}}{2 k T_{0}} \xi^{2}\right]
$$

and $h^{s}$ being the small perturbation with respect to the equilibrium state. In Eq. (4), $n_{0}^{s}$ represents the equilibrium number density and $m^{s}$ denotes the molecular mass. Using Eq. (3), the system of nonstationary BGK-Boltzmann equations reads as

$$
\frac{\partial h^{s}}{\partial t^{\prime}}+c_{z} \frac{\partial h^{s}}{\partial z^{\prime}}=L h_{B G K}^{s} \quad s=1,2
$$

where $L h_{B G K}^{s}$ is the linearized BGK collision operator given by [2]

$$
\begin{gathered}
L h^{1}{ }_{B G K}=v_{1}\left\{\rho^{1}+2\left(1-\Gamma^{1}\right) \mathbf{c} \cdot \mathbf{v}^{1}+2 \Gamma^{1} \mathbf{c} \cdot \mathbf{v}^{2}\right. \\
\left.+\left[1-\frac{2 \Gamma^{1} M_{12}}{\left(1+M_{12}\right)}\right]\left(|\mathbf{c}|^{2}-\frac{3}{2}\right) \tau^{1}+\frac{2 \Gamma^{1} M_{12}}{\left(1+M_{12}\right)}\left(|\mathbf{c}|^{2}-\frac{3}{2}\right) \tau^{2}-h^{1}\right\} \\
\left.+\left[1-\frac{2 \Gamma^{2}}{\left(1+M_{12}\right)}\right]\left(\frac{|\mathbf{c}|^{2}}{M_{12}}-\frac{3}{2}\right) \tau^{2}+\frac{2 \Gamma^{2}}{\left(1+M_{12}\right)}\left(\frac{|\mathbf{c}|^{2}}{M_{12}}-\frac{3}{2}\right) \tau^{1}-h^{2}\right\}
\end{gathered}
$$

In Eqs. (6)-(7), $v_{s}$ are suitable collision frequencies (independent from the molecular velocity $\xi), \mathbf{c}=\frac{\xi}{\sqrt{2 \frac{k}{m^{1}} T_{0}}}, M_{12}=m^{1} / m^{2}, \Gamma^{1}=\frac{v_{1}^{12} \mu^{12}}{v_{1} m^{1}} n_{0}^{2}, \Gamma^{2}=\frac{v_{1}^{12} \mu^{12}}{v_{2} m^{2}} n_{0}^{1}$, where $\mu^{12}=m^{1} m^{2} /\left(m^{1}+m^{2}\right)$ stands for the reduced mass, and $v_{k}^{s r}$ are suitably weighted integrals of the differential cross sections relevant to collisions between particles of species $s$ and $r$ [8]. The dimensionless macroscopic perturbed density $\rho^{s}$, velocity $\mathbf{v}^{s}$, temperature $\tau^{s}$ are obtained by taking the moments of $h^{s}$.

It is convenient now to rescale all variables appearing in Eq. (5) as follows: 


$$
t=t^{\prime} / \theta_{1}, \quad z=z^{\prime} /\left(v_{0}^{1} \theta_{1}\right)
$$

with $\theta_{1}=1 / v_{1}, v_{0}^{1}=\sqrt{2 k T_{0} / m^{1}}$. Furthermore, we define $\Theta_{12}=\theta_{1} / \theta_{2}=v_{2} / v_{1}$ and $\delta=d /\left(v_{0}^{1} \theta_{1}\right)$, which is the dimensionless distance between the channel walls as well as the rarefaction parameter (inverse Knudsen number) of the species $s=1$. Since the problem under consideration is one-dimensional in space, the unknown perturbed distribution functions $h^{s}$, as well as the overall quantities, depend only on the $z$ coordinate. Likewise, we can reduce the dimensionality of the molecularvelocity space by introducing the projection procedure. First, we multiply Eq. (5) by $\frac{1}{\pi} e^{-\left(c_{x}{ }^{2}+c_{y}{ }^{2}\right)}$, when $s=1$, and by $\frac{1}{\left(\pi M_{12}\right)} e^{-\left(c_{x}{ }^{2}+c_{y}{ }^{2}\right) / M_{12}}$, when $s=2$, and we integrate over all $c_{x}$ and $c_{y}$. Then, we multiply Eq. (5) by $\frac{1}{\pi}\left(c_{x}^{2}+c_{y}^{2}-1\right) e^{-\left(c_{x}{ }^{2}+c_{y}{ }^{2}\right)}$, when $s=1$, and by $\frac{1}{\left(\pi M_{12}\right)}\left(\frac{c_{x}^{2}+c_{y}^{2}}{M_{12}}-1\right) e^{-\left(c_{x}{ }^{2}+c_{y}{ }^{2}\right) / M_{12}}$, when $s=2$, and we integrate again over all $c_{x}$ and $c_{y}$. The resulting equations after the projection are

$$
\begin{gathered}
\frac{\partial H^{1}}{\partial t}+c_{z} \frac{\partial H^{1}}{\partial z}+H^{1}=\rho^{1}+2\left(1-\Gamma^{1}\right) c_{z} v_{z}^{1}+2 \Gamma^{1} c_{z} v_{z}^{2} \\
+\left[1-\frac{2 \Gamma^{1} M_{12}}{\left(1+M_{12}\right)}\right]\left(c_{z}^{2}-\frac{1}{2}\right) \tau^{1}+\frac{2 \Gamma^{1} M_{12}}{\left(1+M_{12}\right)}\left(c_{z}^{2}-\frac{1}{2}\right) \tau^{2} \\
\frac{\partial H^{2}}{\partial t}+c_{z} \frac{\partial H^{2}}{\partial z}+\Theta_{12} H^{2}=\Theta_{12}\left\{\rho^{2}+\frac{2\left(1-\Gamma^{2}\right)}{M_{12}} c_{z} v_{z}^{2}+\frac{2 \Gamma_{12}^{2}}{M_{1}} v_{z}^{1}\right. \\
\left.+\left[1-\frac{2 \Gamma^{2}}{\left(1+M_{12}\right)}\right]\left(\frac{c_{z}^{2}}{M_{12}}-\frac{1}{2}\right) \tau^{2}+\frac{2 \Gamma^{2}}{\left(1+M_{12}\right)}\left(\frac{c_{z}^{2}}{M_{12}}-\frac{1}{2}\right) \tau^{1}\right\} \\
\frac{\partial \Psi^{1}}{\partial t}+c_{z} \frac{\partial \Psi^{1}}{\partial z}+\Psi^{1}=\left[1-\frac{2 \Gamma^{1} M_{12}}{\left(1+M_{12}\right)}\right] \tau^{1}+\frac{2 \Gamma^{1} M_{12}}{\left(1+M_{12}\right)} \tau^{2} \\
\frac{\partial \Psi^{2}}{\partial t}+c_{z} \frac{\partial \Psi^{2}}{\partial z}+\Theta_{12} \Psi^{2}=\Theta_{12}\left\{\left[1-\frac{2 \Gamma^{2}}{\left(1+M_{12}\right)}\right] \tau^{2}+\frac{2 \Gamma^{2}}{\left(1+M_{12}\right)} \tau^{1}\right\}
\end{gathered}
$$

where the reduced unknown distribution functions $H^{s}$ and $\Psi^{s}$ are defined as

$$
\begin{gathered}
H^{1}\left(z, c_{z}, t\right)=\frac{1}{\pi} \int_{-\infty}^{+\infty} \int_{-\infty}^{+\infty} h^{1}(z, \mathbf{c}, t) e^{-\left(c_{x}^{2}+c_{y}^{2}\right)} d c_{x} d c_{y} \\
H^{2}\left(z, c_{z}, t\right)=\frac{1}{\pi M_{12}} \int_{-\infty}^{+\infty} \int_{-\infty}^{+\infty} h^{2}(z, \mathbf{c}, t) e^{-\left(c_{x}^{2}+c_{y}^{2}\right) / M_{12}} d c_{x} d c_{y} \\
\Psi^{1}\left(z, c_{z}, t\right)=\frac{1}{\pi} \int_{-\infty}^{+\infty} \int_{-\infty}^{+\infty}\left(c_{x}^{2}+c_{y}^{2}-1\right) h^{1}(z, \mathbf{c}, t) e^{-\left(c_{x}^{2}+c_{y}^{2}\right)} d c_{x} d c_{y} \\
\Psi^{2}\left(z, c_{z}, t\right)=\frac{1}{\pi M_{12}} \int_{-\infty}^{+\infty} \int_{-\infty}^{+\infty}\left(\frac{c_{x}^{2}+c_{y}^{2}}{M_{12}}-1\right) h^{2}(z, \mathbf{c}, t) e^{-\left(c_{x}^{2}+c_{y}^{2}\right) / M_{12}} d c_{x} d c_{y}
\end{gathered}
$$


The macroscopic fields appearing on the right-hand side of Eqs. (9)-(12) are given by

$$
\begin{gathered}
\rho^{1}(z, t)=\frac{1}{\sqrt{\pi}} \int_{-\infty}^{+\infty} H^{1} e^{-c_{z}^{2}} d c_{z} \\
\rho^{2}(z, t)=\frac{1}{\sqrt{\pi M_{12}}} \int_{-\infty}^{+\infty} H^{2} e^{-c_{z}^{2} / M_{12}} d c_{z} \\
v_{z}^{1}(z, t)=\frac{1}{\sqrt{\pi}} \int_{-\infty}^{+\infty} c_{z} H^{1} e^{-c_{z}^{2}} d c_{z} \\
v_{z}^{2}(z, t)=\frac{1}{\sqrt{\pi M_{12}}} \int_{-\infty}^{+\infty} c_{z} H^{2} e^{-c_{z}^{2} / M_{12}} d c_{z} \\
\tau^{1}(z, t)=\frac{1}{\sqrt{\pi}} \int_{-\infty}^{+\infty} \frac{2}{3}\left[\left(c_{z}^{2}-1 / 2\right) H^{1}+\Psi^{1}\right] e^{-c_{z}^{2}} d c_{z} \\
\tau^{2}(z, t)=\frac{1}{\sqrt{\pi M_{12}}} \int_{-\infty}^{+\infty} \frac{2}{3}\left[\left(\frac{c_{z}^{2}}{M_{12}}-\frac{1}{2}\right) H^{2}+\Psi^{2}\right] e^{-c_{z}^{2} / M_{12}} d c_{z}
\end{gathered}
$$

Applying the same projection procedure to the linearized boundary conditions reported in [9] (Eqs. (17)-(18)) describing the diffuse scattering of gaseous particles on both walls of the microchannel, we are led to derive the following expressions

$$
\begin{array}{cc}
H^{1}\left(z=-\delta / 2, c_{z}, t\right)=\left(\sqrt{\pi}+2 c_{z}\right) U_{w} & \\
-2 \int_{\tilde{c}_{z}<0} d \tilde{c}_{z} \tilde{c}_{z} e^{-\tilde{c}_{z}^{2}} H^{1}\left(z=-\delta / 2, \tilde{c}_{z}, t\right) & c_{z}>0 \\
H^{2}\left(z=-\delta / 2, c_{z}, t\right)=\left(\sqrt{\frac{\pi}{M_{12}}}+\frac{2 c_{z}}{M_{12}}\right) U_{w} & \\
-\frac{2}{M_{12}} \int_{\tilde{c}_{z}<0} d \tilde{c}_{z} \tilde{c}_{z} e^{-\tilde{c}_{z}^{2} / M_{12}} H^{2}\left(z=-\delta / 2, \tilde{c}_{z}, t\right) & c_{z}>0 \\
\Psi^{1}\left(z=-\delta / 2, c_{z}, t\right)=\Psi^{2}\left(z=-\delta / 2, c_{z}, t\right)=0 & c_{z}>0 \\
H^{1}\left(z=\delta / 2, c_{z}, t\right)=2 \int_{\tilde{c}_{z}>0} d \tilde{c}_{z} \tilde{c}_{z} e^{-\tilde{c}_{z}^{2}} H^{1}\left(z=\delta / 2, \tilde{c}_{z}, t\right) & c_{z}<0 \\
H^{2}\left(z=\delta / 2, c_{z}, t\right)=\frac{2}{M_{12}} \int_{\tilde{c}_{z}>0} d \tilde{c}_{z} \tilde{c}_{z} e^{-\tilde{c}_{z}^{2} / M_{12}} H^{2}\left(z=\delta / 2, \tilde{c}_{z}, t\right) & c_{z}<0 \\
\Psi^{1}\left(z=\delta / 2, c_{z}, t\right)=\Psi^{2}\left(z=\delta / 2, c_{z}, t\right)=0 & c_{z}<0
\end{array}
$$

In Eqs. (23) and (24), $U_{w}$ is the dimensionless wall velocity given by

$$
U_{w}(t)=U_{0} \sin (\omega t)
$$


with $U_{w}=U_{w}^{\prime} / v_{0}^{1}, U_{0}=U_{0}^{\prime} / v_{0}^{1}, \omega=\theta_{1} \omega^{\prime}, T=2 \pi / \omega=T^{\prime} / \theta_{1}$. The time-dependent problem described by Eqs. (9)-(12), with boundary conditions given by Eqs. (23)(28), has been numerically solved by a deterministic finite-difference method. The region between the plates has been divided into a number of cells of the same size. For this particular application, high spatial resolution is required in order to capture properly the propagation of sound waves with short wavelength. It should be noted that, following previous numerical studies (see [18]), the emitter plate displacement is not taken into account. The gas feels the plate motion only through the boundary conditions (23)-(28). Similarly, a regular net of nodes has been arranged into a finite domain of the one-dimensional velocity space, large enough to contain the significant part of the distribution functions at any spatial location. In general, the extent of the velocity distribution function of species $s(s=1,2)$ in the molecular velocity space is inversely proportional to $\sqrt{m^{s}}$. Thus, a separate grid for each species should be considered. Indeed, due to the normalization adopted in the present paper, we were able to use only one grid in the velocity space since the scaling factor: $\sqrt{m^{1} / m^{2}}=\sqrt{M_{12}}$ (which allows to switch between the grid associated with the first species and that associated with the second species) appears directly in the equations defining the reduced distribution functions $H^{s}$ and $\Psi^{s}$ (Eqs. (13)(16)) and the macroscopic fields (Eqs. (17)-(22)). Then, the distribution functions are assumed to be constant within each cell of the phase space. Further details on the standard (upwind) implicit finite-difference scheme employed in the present computations are reported in [18]. The convergence of the numerical results presented has been extensively examined by modifying the number of grid points chosen in phase space. The variation of the components of the macroscopic fields due to the increase of the grid parameters was the criterion of the calculation accuracy. In addition, it has been checked that, allowing our data to be reduced to the case of a single monoatomic gas by taking $m^{1}=m^{2}$, the results presented in [9] can be recovered.

In order to investigate more deeply the influence of the parameters peculiar to a binary gaseous mixture on the sound wave propagation, we present in Sections 3 and 4 two semi-analytical methods of solution for the BGK-Boltzmann equations (9)(12), which retain their usefulness since they are capable of providing an analytical representation of the solution and hence a picture of its qualitative behavior.

\section{Integral Equations}

Since the vibrations of the lower wall of the microchannel are generated by a timeharmonic forcing (of frequency $\omega$ ) of the form $\sin (\omega t)$ (see Eq. (29)), we introduce the following expression $U_{w}=U_{0} e^{i \omega t}$ in Eqs. (23) and (24) and then we look for solutions of Eqs. (9)-(12) under the form

$$
\begin{aligned}
& H^{s}\left(z, c_{z}, t\right)=\mathscr{H}^{s}\left(z, c_{z}\right) e^{i \omega t} \quad s=1,2 \\
& \Psi^{s}\left(z, c_{z}, t\right)=\Psi^{s}\left(z, c_{z}\right) e^{i \omega t} \quad s=1,2 .
\end{aligned}
$$


The solutions of the original problem are then recovered by taking the imaginary part of $H^{s}$ and $\Psi^{s}$. Putting Eqs. (30) and (31) into Eqs. (9)-(12) and then integrating the resulting equations along the trajectories of the molecules, we obtain an explicit expression for the distribution functions

$$
\begin{aligned}
& \mathscr{H}^{1}\left(z, c_{z}\right)=e^{-\frac{z}{c_{z}}(1+i \omega)} e^{-\frac{\delta \operatorname{sgn} c_{z}}{2 c_{z}}(1+i \omega)} \mathscr{H}^{1}\left(-\delta / 2 \operatorname{sgn} c_{z}, c_{z}\right) \\
& +\int_{-\delta / 2 \operatorname{sgn} c_{z}}^{z} d s c_{z}^{-1}\left\{\rho^{1}(s)+2\left(1-\Gamma^{1}\right) c_{z} \mathrm{v}_{z}^{1}(s)+2 \Gamma^{1} c_{z} \mathrm{v}_{z}^{2}(s)\right. \\
& \left.+\left[1-\frac{2 \Gamma^{1} M_{12}}{\left(1+M_{12}\right)}\right]\left(c_{z}^{2}-\frac{1}{2}\right) \tau^{1}(s)+\frac{2 \Gamma^{1} M_{12}}{\left(1+M_{12}\right)}\left(c_{z}^{2}-\frac{1}{2}\right) \tau^{2}(s)\right\} \\
& \times e^{-|z-s| \frac{(1+i \omega)}{\left|c_{z}\right|}} \\
& \mathscr{H}^{2}\left(z, c_{z}\right)=e^{-\frac{z}{c_{z}}\left(\Theta_{12}+i \omega\right)} e^{-\frac{\delta \operatorname{sgn} c_{z}}{2 c_{z}}\left(\Theta_{12}+i \omega\right)} \mathscr{H}^{2}\left(-\delta / 2 \operatorname{sgn} c_{z}, c_{z}\right) \\
& +\int_{-\delta / 2 \operatorname{sgn} c_{z}}^{z} d s \frac{\Theta_{12}}{c_{z}}\left\{\rho^{2}(s)+\frac{2\left(1-\Gamma^{2}\right)}{M_{12}} c_{z} \mathrm{v}_{z}^{2}(s)+\frac{2 \Gamma^{2}}{M_{12}} c_{z} \mathrm{v}_{z}^{1}(s)\right. \\
& \left.+\left[1-\frac{2 \Gamma^{2}}{\left(1+M_{12}\right)}\right]\left(\frac{c_{z}^{2}}{M_{12}}-\frac{1}{2}\right) \tau^{2}(s)+\frac{2 \Gamma^{2}}{\left(1+M_{12}\right)}\left(\frac{c_{z}^{2}}{M_{12}}-\frac{1}{2}\right) \tau^{1}(s)\right\} \\
& \times e^{-|z-s| \frac{\left(\Theta_{12}+i \omega\right)}{\left|c_{z}\right|}} \\
& \Psi^{1}\left(z, c_{z}\right)=\int_{-\delta / 2 \operatorname{sgn} c_{z}}^{z} d s c_{z}^{-1}\left\{\left[1-\frac{2 \Gamma^{1} M_{12}}{\left(1+M_{12}\right)}\right] \tau^{1}(s)\right. \\
& \left.+\frac{2 \Gamma^{1} M_{12}}{\left(1+M_{12}\right)} \tau^{2}(s)\right\} \times e^{-|z-s| \frac{(1+i \omega)}{\left|c_{z}\right|}} \\
& \Psi^{2}\left(z, c_{z}\right)=\int_{-\delta / 2 \operatorname{sgn} c_{z}}^{z} d s \frac{\Theta_{12}}{c_{z}}\left\{\left[1-\frac{2 \Gamma^{2}}{\left(1+M_{12}\right)}\right] \tau^{2}(s)\right. \\
& \left.+\frac{2 \Gamma^{2}}{\left(1+M_{12}\right)} \tau^{1}(s)\right\} \times e^{-|z-s| \frac{\left(\Theta_{12}+i \omega\right)}{\left|c_{z}\right|}}
\end{aligned}
$$

Inserting in the definitions (17)-(22) the distribution functions (32)-(35), together with the boundary conditions, the integral equations for the macroscopic fields $\rho^{s}$, 
$\nu_{z}^{s}, \tau^{s}$ can be explicitly obtained. To solve the resulting system of six coupled integral equations, one can extend a finite difference technique first introduced in a paper by Cercignani and Daneri [5], by using the method reported in [7], [9]. In view of our subsequent considerations, we are interested in writing down the integral equations for the bulk velocities of the gas components:

$$
\begin{aligned}
& \mathrm{v}_{z}^{1}(z)=\mathscr{K}_{1}(z, \gamma)+\frac{1}{\sqrt{\pi}} \int_{-\delta / 2}^{\delta / 2} d s \rho^{1}(s) \operatorname{sgn}(z-s) T_{0}(|z-s| \gamma) \\
& -\frac{1}{\sqrt{\pi}} \int_{-\delta / 2}^{\delta / 2} d s \rho^{1}(s) \mathscr{K}_{2}(z, s, \gamma) \\
& +\frac{2}{\sqrt{\pi}} \int_{-\delta / 2}^{\delta / 2} d s\left[\left(1-\Gamma^{1}\right) \mathrm{v}_{z}^{1}(s)+\Gamma^{1} \mathrm{v}_{z}^{2}(s)\right] T_{1}(|z-s| \gamma) \\
& -\frac{1}{\sqrt{\pi}} \int_{-\delta / 2}^{\delta / 2} d s\left[\left(1-\Gamma^{1}\right) \mathrm{v}_{z}^{1}(s)+\Gamma^{1} \mathrm{v}_{z}^{2}(s)\right] \mathscr{K}_{3}(z, s, \gamma) \\
& +\frac{1}{\sqrt{\pi}} \int_{-\delta / 2}^{\delta / 2} d s\left[\left(1-\frac{2 \Gamma^{1} M_{12}}{\left(1+M_{12}\right)}\right) \tau^{1}(s)+\frac{2 \Gamma^{1} M_{12}}{\left(1+M_{12}\right)} \tau^{2}(s)\right] \\
& \times \operatorname{sgn}(z-s)\left[T_{2}(|z-s| \gamma)-\frac{1}{2} T_{0}(|z-s| \gamma)\right] \\
& -\frac{4}{\sqrt{\pi}} \int_{-\delta / 2}^{\delta / 2} d s\left[\left(1-\frac{2 \Gamma^{1} M_{12}}{\left(1+M_{12}\right)}\right) \tau^{1}(s)+\frac{2 \Gamma^{1} M_{12}}{\left(1+M_{12}\right)} \tau^{2}(s)\right] \mathscr{K}_{4}(z, s, \gamma) \\
& \mathrm{v}_{z}^{2}(z)=\mathscr{K}_{1}(z, \tilde{\gamma})+\frac{\Theta_{12}}{\sqrt{\pi}} \int_{-\delta / 2}^{\delta / 2} d s \rho^{2}(s) \operatorname{sgn}(z-s) T_{0}(|z-s| \tilde{\gamma}) \\
& -\frac{\Theta_{12}}{\sqrt{\pi}} \int_{-\delta / 2}^{\delta / 2} d s \rho^{2}(s) \mathscr{K}_{2}(z, s, \tilde{\gamma}) \\
& +\frac{2 \Theta_{12}}{\sqrt{\pi M_{12}}} \int_{-\delta / 2}^{\delta / 2} d s\left[\left(1-\Gamma^{2}\right) \mathrm{v}_{z}^{2}(s)+\Gamma^{2} \mathrm{v}_{z}^{1}(s)\right] T_{1}(|z-s| \tilde{\gamma}) \\
& -\frac{\Theta_{12}}{\sqrt{\pi M_{12}}} \int_{-\delta / 2}^{\delta / 2} d s\left[\left(1-\Gamma^{2}\right) \mathrm{v}_{z}^{2}(s)+\Gamma^{2} \mathrm{v}_{z}^{1}(s)\right] \mathscr{K}_{3}(z, s, \tilde{\gamma}) \\
& +\frac{\Theta_{12}}{\sqrt{\pi}} \int_{-\delta / 2}^{\delta / 2} d s\left[\left(1-\frac{2 \Gamma^{2}}{\left(1+M_{12}\right)}\right) \tau^{2}(s)+\frac{2 \Gamma^{2}}{\left(1+M_{12}\right)} \tau^{1}(s)\right] \\
& \times \operatorname{sgn}(z-s)\left[T_{2}(|z-s| \tilde{\gamma})-\frac{1}{2} T_{0}(|z-s| \tilde{\gamma})\right] \\
& -\frac{4 \Theta_{12}}{\sqrt{\pi}} \int_{-\delta / 2}^{\delta / 2} d s\left[\left(1-\frac{2 \Gamma^{2}}{\left(1+M_{12}\right)}\right) \tau^{2}(s)+\frac{2 \Gamma^{2}}{\left(1+M_{12}\right)} \tau^{1}(s)\right] \mathscr{K}_{4}(z, s, \tilde{\gamma})
\end{aligned}
$$

where $\gamma=(1+i \omega), \tilde{\gamma}=\left(\Theta_{12}+i \omega\right) / \sqrt{M_{12}}$, and the symbols $\mathscr{K}_{i}(i=1, \ldots, 4)$ stand for expressions involving products of the Abramowitz functions $T_{n}$ defined by 


$$
T_{n}(x):=\int_{0}^{+\infty} s^{n} e^{-s^{2}-x / s} d s .
$$

The explicit form of the functions $\mathscr{K}_{i}$ is given in [2]. In Section 5, we will show that some basic properties of sound wave propagation in different binary mixtures can be deduced simply by a qualitative analysis of the integral equations (36)-(37).

\section{The Elementary Solutions}

In order to simplify the numerical procedure needed to solve our problem, one can even improve the ansatz (30)-(31) through a separation of spatial and molecular velocity variables. This can be done by taking advantage of the explicit form of the distribution functions $\left(H^{s}, \Psi^{s}\right)$ found in Section 3.

The four coupled balance equations (9)-(12) can be rewritten in matrix notation as follows

$$
\frac{\partial}{\partial t} \mathbf{Z}\left(z, c_{z}, t\right)+c_{z} \frac{\partial}{\partial z} \mathbf{Z}\left(z, c_{z}, t\right)+\Sigma \mathbf{Z}\left(z, c_{z}, t\right)=\Sigma \int_{-\infty}^{+\infty} \mathrm{K}\left(c_{z}, c_{z}^{\prime}\right) \mathbf{Z}\left(z, c_{z}^{\prime}, t\right) d c_{z}^{\prime}
$$

where $\mathbf{Z}\left(z, c_{z}, t\right)=\left(H^{1}, \Psi^{1}, H^{2}, \Psi^{2}\right)^{T}$ and $\Sigma=\operatorname{diag}\left(1,1, \Theta_{12}, \Theta_{12}\right)$. In addition, the components $k_{i, j}\left(c_{z}, c_{z}^{\prime}\right)$ of the kernel $\mathrm{K}\left(c_{z}, c_{z}^{\prime}\right)$ are listed explicitly in Appendix 1. The boundary conditions associated with Eq. (39) can be likewise expressed in the following form

$$
\begin{gathered}
\mathbf{Z}\left(z=-\delta / 2, c_{z}, t\right)=\mathbf{R} U_{w}-2 \int_{\tilde{c}_{z}<0} d \tilde{c}_{z} \tilde{c}_{z} \mathrm{DZ}\left(z=-\delta / 2, \tilde{c}_{z}, t\right) \quad c_{z}>0 \\
\mathbf{Z}\left(z=\delta / 2, c_{z}, t\right)=2 \int_{\tilde{c}_{z}>0} d \tilde{c}_{z} \tilde{c}_{z} \mathrm{D} \mathbf{Z}\left(z=\delta / 2, \tilde{c}_{z}, t\right) \quad c_{z}<0
\end{gathered}
$$

where $\mathbf{R}=\left(\left(\sqrt{\pi}+2 c_{z}\right), 0,\left(\sqrt{\frac{\pi}{M_{12}}}+\frac{2 c_{z}}{M_{12}}\right), 0\right)^{T}$ and $\mathrm{D}=\operatorname{diag}\left(\mathrm{e}^{-\tilde{\mathrm{c}}_{\mathrm{z}}^{2}}, 0, \frac{1}{\mathrm{M}_{12}} \mathrm{e}^{-\tilde{\mathrm{c}}_{\mathrm{z}}^{2} / \mathrm{M}_{12}}, 0\right)$.

We seek solutions of Eq. (39) of the form

$$
\mathbf{Z}\left(z, c_{z}, t\right)=e^{s t} e^{-(s 1+\Sigma) \frac{z}{v}} \Phi\left(v, c_{z}\right) \quad \text { with } s=\mathrm{i} \omega
$$

where the separation constants $v$ and the elementary solutions $\Phi\left(v, c_{z}\right)$ (in general complex) are to be determined. Substituting Eq. (42) into Eq. (39), we find

$$
\begin{aligned}
\left(v-c_{z}\right)(s 1+\Sigma) \Phi\left(v, c_{z}\right)= & v \Sigma \int_{0}^{+\infty}\left[\mathrm{K}\left(c_{z}^{\prime}, c_{z}\right) \Phi\left(v, c_{z}^{\prime}\right)\right. \\
& \left.+\mathrm{K}\left(-c_{z}^{\prime}, c_{z}\right) \Phi\left(v,-c_{z}^{\prime}\right)\right] d c_{z}^{\prime}
\end{aligned}
$$


From Eq. (43), by replacing $c_{z} \rightarrow-c_{z}$, one has

$$
\begin{aligned}
\left(v+c_{z}\right)(s 1+\Sigma) \Phi\left(v,-c_{z}\right)= & v \Sigma \int_{0}^{+\infty}\left[\mathrm{K}\left(c_{z}^{\prime},-c_{z}\right) \Phi\left(v, c_{z}^{\prime}\right)\right. \\
& \left.+\mathrm{K}\left(-c_{z}^{\prime},-c_{z}\right) \Phi\left(v,-c_{z}^{\prime}\right)\right] d c_{z}^{\prime}
\end{aligned}
$$

Now, adding and subtracting Eqs. (43)-(44), one from the other, and taking into account the following identities

$$
\begin{aligned}
& \mathrm{K}\left(c_{z}^{\prime},-c_{z}\right)=\mathrm{K}\left(-c_{z}^{\prime}, c_{z}\right) \\
& \mathrm{K}\left(-c_{z}^{\prime},-c_{z}\right)=\mathrm{K}\left(c_{z}^{\prime}, c_{z}\right)
\end{aligned}
$$

we conclude

$$
\frac{1}{c_{z}^{2}}\left[\mathbf{V}\left(v, c_{z}\right)-(s 1+\Sigma)^{-1} \Sigma \int_{0}^{+\infty} \Gamma\left(c_{z}^{\prime}, c_{z}\right) \mathbf{V}\left(v, c_{z}^{\prime}\right) d c_{z}^{\prime}\right]=\lambda \mathbf{V}\left(v, c_{z}\right)
$$

and

$$
\mathbf{U}\left(v, c_{z}\right)=\frac{v}{c_{z}}\left[\mathbf{V}\left(v, c_{z}\right)-(s 1+\Sigma)^{-1} \Sigma \int_{0}^{+\infty} \mathrm{K}_{-}\left(c_{z}^{\prime}, c_{z}\right) \mathbf{V}\left(v, c_{z}^{\prime}\right) d c_{z}^{\prime}\right]
$$

where

$$
\begin{gathered}
\mathbf{U}\left(v, c_{z}\right)=\Phi\left(v, c_{z}\right)+\Phi\left(v,-c_{z}\right) \\
\mathbf{V}\left(v, c_{z}\right)=\Phi\left(v, c_{z}\right)-\Phi\left(v,-c_{z}\right) \\
\lambda=\frac{1}{v^{2}} \\
\mathrm{~K}_{+}\left(c_{z}^{\prime}, c_{z}\right)=\mathrm{K}\left(c_{z}^{\prime}, c_{z}\right)+\mathrm{K}\left(-c_{z}^{\prime}, c_{z}\right) \\
\mathrm{K}_{-}\left(c_{z}^{\prime}, c_{z}\right)=\mathrm{K}\left(c_{z}^{\prime}, c_{z}\right)-\mathrm{K}\left(-c_{z}^{\prime}, c_{z}\right) \\
\Gamma\left(c_{z}^{\prime}, c_{z}\right)=\mathrm{K}_{-}\left(c_{z}^{\prime}, c_{z}\right)+\frac{c_{z}}{c_{z}^{\prime}} \mathrm{K}_{+}\left(c_{z}^{\prime}, c_{z}\right) \\
-\int_{0}^{+\infty} \frac{c_{z}}{c_{z}^{\prime \prime}} \mathrm{K}_{+}\left(c_{z}^{\prime \prime}, c_{z}\right)(s 1+\Sigma)^{-1} \Sigma \mathrm{K}_{-}\left(c_{z}^{\prime}, c_{z}^{\prime \prime}\right) d c_{z}^{\prime \prime}
\end{gathered}
$$


Equation (47) defines our eigenvalue problem. Once this eigenvalue problem is solved, we have the elementary solutions from

$$
\begin{gathered}
\Phi\left(v, c_{z}\right)=\frac{1}{2}\left[\mathbf{U}\left(v, c_{z}\right)+\mathbf{V}\left(v, c_{z}\right)\right] \\
\Phi\left(v,-c_{z}\right)=\frac{1}{2}\left[\mathbf{U}\left(v, c_{z}\right)-\mathbf{V}\left(v, c_{z}\right)\right]
\end{gathered}
$$

Then, by taking into account the completeness theorems stated in [4], the general solution of Eq. (39) can be written as follows

$$
\begin{aligned}
\mathbf{Z}\left(z, c_{z}, t\right)=e^{s t} & \int_{0}^{+\infty} A(v)\left\{\Phi\left(v, c_{z}\right) e^{-(s 1+\Sigma)\left[\frac{z}{v}+\frac{\delta}{2 v}\right]}\right. \\
& \left.+\Phi\left(v,-c_{z}\right) e^{-(s 1+\Sigma)\left[-\frac{z}{v}+\frac{\delta}{2 v}\right]}\right\} d v
\end{aligned}
$$

where the symmetry condition

$$
\Phi\left(v, c_{z}\right)=\Phi\left(-v,-c_{z}\right)
$$

has been exploited. In Eq. (57) the arbitrary constant $A(v)$, to be determined from the boundary conditions, has been redefined by inserting a factor

$$
e^{-(s 1+\Sigma) \frac{\delta}{2|v|}}
$$

according to the explicit form of the distribution functions found in Section 3. In order to solve numerically Eqs. (47), (48), (55), (56), (57), one can introduce a half-range quadrature scheme as done by Siewert et al. [24], [23], [10]. The algorithm requires only an eigenvalue/eigenvector routine and the solution of a system of linear algebraic equations. This allows to greatly reduce the computational effort if compared, for instance, with that required to solve a system of coupled integral equations as presented in Section 3. An alternative approach to handle numerically time-dependent problems in terms of elementary solutions has been recently proposed by Gosse in [11]. His scheme requires first the computation of the elementary solutions of the associated stationary boundary-value problem (which are always real functions) and then, in order to recover the time evolution, a well-balanced Godunov scheme is implemented.

\section{Results and Discussion}

The results presented in the following and obtained through a numerical integration of Eqs. (9)-(12) refer to the noble gaseous mixtures of He-Xe (that is, helium with molecular mass $m^{1}=4.0026 \mathrm{au}$ and xenon with molecular mass $m^{2}=131.29 \mathrm{au}$ ) 

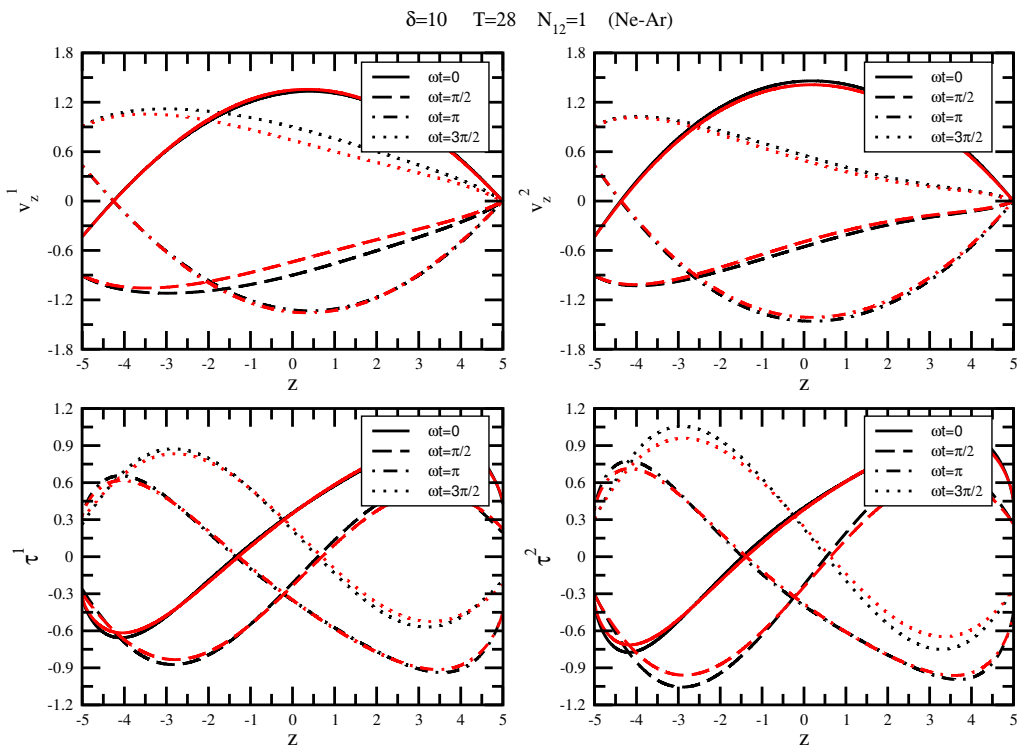

Fig. 1 Variation of the macroscopic velocities and temperatures of the $\mathrm{Ne}-\mathrm{Ar}$ mixture components (with the same molar concentrations), in the $z$-direction across the gap of the channel for $\delta=10$ and $T=28$. Comparison between the BGK model (black lines) and the McCormack model (red lines). In each panel, the profiles of the macroscopic quantities are shown at different stages during a period of oscillation of the moving wall.

and $\mathrm{Ne}-\mathrm{Ar}$ (that is, neon with molecular mass $m^{1}=20.179 \mathrm{au}$ and argon with molecular mass $m^{2}=39.948 \mathrm{au}$ ) with the same ratio of the molar concentrations of the two species $N_{12}=n_{0}^{1} / n_{0}^{2}=1$. In order to inspect the physical processes going on in both gas mixtures, we are led to examine the macroscopic profiles of the mixture components in different regimes.

\subsection{Near-Continuum Regime}

We report in Figures 1-2 the velocity and temperature profile of the mixture components as a function of the distance across the gap of the channel (at different stages during a period of oscillation) when $\delta=10$. In order to prove the reliability of the results obtained from a numerical implementation of the BGK model presented in the present paper, we have also included in these pictures the outcomes derived from a numerical solution of the linearized McCormack model, widely considered in the literature to describe a gaseous mixture [23], [24]. For the sake of completeness, this model is briefly described in Appendix 2. In all the figures presented in the following, the lightest component of each mixture has been labelled with superscript 1 , while the heaviest one with superscript 2 . 

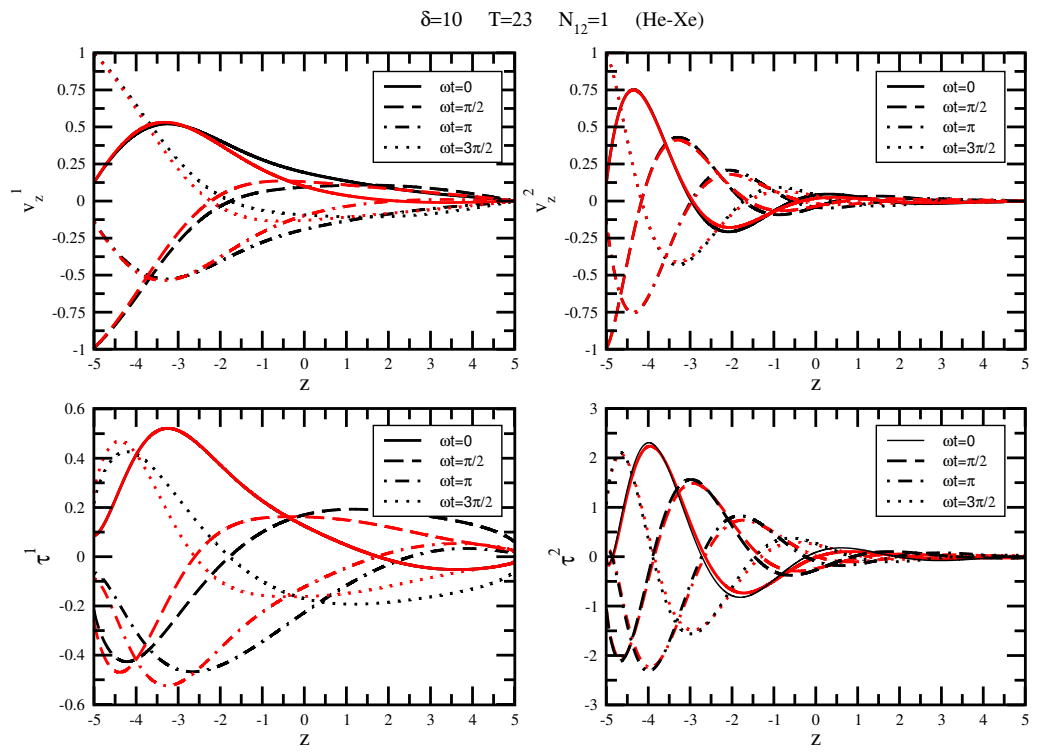

Fig. 2 Variation of the macroscopic velocities and temperatures of the He-Xe mixture components (with the same molar concentrations), in the $z$-direction across the gap of the channel for $\delta=10$ and $T=23$. Comparison between the BGK model (black lines) and the McCormack model (red lines). In each panel, the profiles of the macroscopic quantities are shown at different stages during a period of oscillation of the moving wall.

In the case of the Ne-Ar mixture, both species have the same macroscopic fields (see Fig. 1), while for the He-Xe mixture, the pictures reveal that two different forced-sound modes are simultaneously present: a fast and a slow wave (see Fig. 2). The slow wave is a damped soundlike mode primarily carried by the Xe, while the fast wave should be associated to the He-component of the mixture. Looking at Figures 3 and 4, where the velocity and temperature profiles of the components of the He-Xe mixture are shown for different values of the period $T$ ( $T=2$ and $T=70$ ), one can infer that the characteristics of the macroscopic fields, associated with the two sound modes, depend strongly on the excitation frequency of the modes themselves. At $T=2$, both modes are damped, then, when $T$ increases, they tend to equilibrate, even if, in a neighborhood of $T=23$, the sound mode associated to the heaviest component of the mixture appears more damped than the other and with a very different speed of propagation. To explain quantitatively the different behavior of the two gaseous mixtures in terms of the parameters peculiar to a mixture itself, we can resort to an analysis of the integral equations given by Eqs. (36) and (37). In the near-continuum regime ( $\delta \geq 10)$, the integrals in Eqs. (36) and (37) give the main contribution to the velocities of gas components. For mixtures like $\mathrm{Ne}-\mathrm{Ar}$, whose constituents have comparable molecular mass, such that $\gamma \simeq \tilde{\gamma}, \Theta_{12} \simeq 1$, and $M_{12} \simeq 1$, the velocity profiles of the two species are symmetric by interchanging superscripts 1 and 2 (as the results reported in Fig. 1 show). In this case, Eq. (36) 

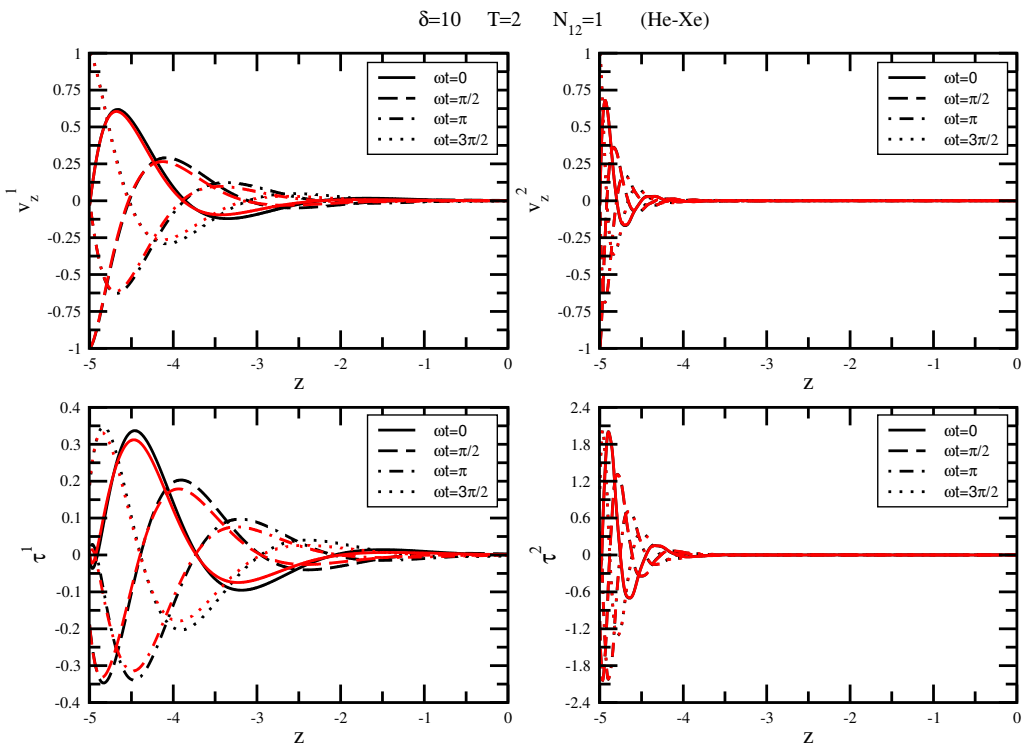

Fig. 3 Variation of the macroscopic velocities and temperatures of the $\mathrm{He}-\mathrm{Xe}$ mixture components (with the same molar concentrations), in the $z$-direction across the gap of the channel for $\delta=10$ and $T=2$. Comparison between the BGK model (black lines) and the McCormack model (red lines). In each panel, the profiles of the macroscopic quantities are shown at different stages during a period of oscillation of the moving wall. In this figure we report only half of the spatial range since for $z \in[0,5]$ both modes are completely damped and identically equal to zero.

reduces to the equation for the velocity field of a single-component gas [9], since the terms $\left(-\Gamma^{1} \mathrm{v}_{z}^{1}\right)$ and $\left(\Gamma^{1} \mathrm{v}_{z}^{2}\right)$ cancel out as well as the terms: $-\left[\frac{2 \Gamma^{1} M_{12}}{\left(1+M_{12}\right)}\right] \tau^{1}$ and $\left[\frac{2 \Gamma^{1} M_{12}}{\left(1+M_{12}\right)}\right] \tau^{2}$. On the contrary, for mixtures like $\mathrm{He}-\mathrm{Xe}$, whose constituents have very different molecular mass, such that $\Theta_{12}<<1, M_{12}<<1$, and the real and imaginary parts of $\tilde{\gamma}$ are higher than those of $\gamma$, the field $v_{z}^{2}$, described only by the Abramowitz functions which depend on $\tilde{\gamma}$, approaches zero more quickly, as function of $z$, than $\mathrm{v}_{z}^{1}$. This is true for each value of $T$. By comparing Eq. (36), when $M_{12} \simeq 0$, with the integral equation for the macroscopic velocity field of a single-component gas, reported in [9] (Eq. (67)), we infer that the velocity field of the lightest species $\mathrm{v}_{z}^{1}$ is the same as that reported in [9] except that, in Eq. (36), the influence of the integral term involving $\mathrm{v}_{z}^{1}$ is weighted by the factor $\left(1-\Gamma^{1}\right)$ instead of 1 . This means that the wave associated to the He-component is not a single gasbased sound mode. Only when $\Gamma^{1} \rightarrow 0$, that is, for $N_{12}>>1$, Eq. (36) reduces to the equation for the velocity field of a single-component gas. 

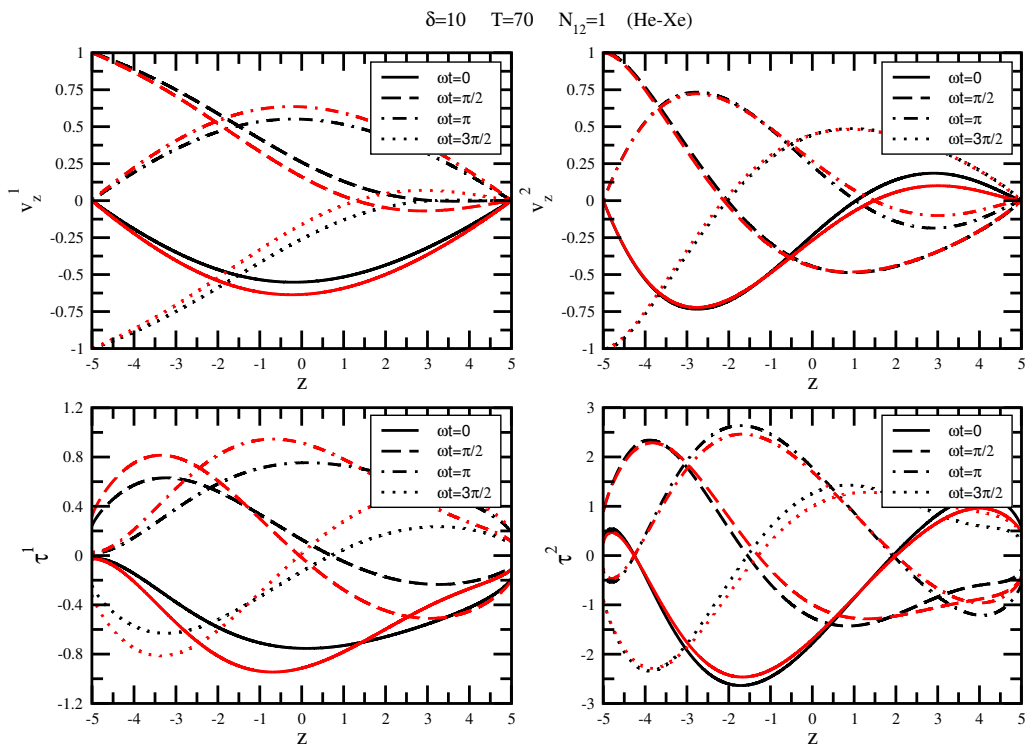

Fig. 4 Variation of the macroscopic velocities and temperatures of the He-Xe mixture components (with the same molar concentrations), in the $z$-direction across the gap of the channel for $\delta=10$ and $T=70$. Comparison between the BGK model (black lines) and the McCormack model (red lines). In each panel, the profiles of the macroscopic quantities are shown at different stages during a period of oscillation of the moving wall.

\subsection{Near-Free Molecular Flow Regime and Transitional Region}

In the near-free molecular flow regime $(\delta=0.1)$, the macroscopic field profiles of the lightest species of both mixtures coincide with those obtained for a single gas [9] (see Figures 5-6). In the case of the Ne-Ar mixture, the velocity and temperature profiles of the two gas components are similar in shape, even if quantitatively different (see Fig. 5). In the case of the He-Xe mixture, Fig. 6 reveals that, for $\delta=0.1$, two different forced-sound modes are simultaneously present: a fast and a slow wave, with the same properties already analyzed in Subsection 5.1. The only difference with the near-continuum case is that, for $\delta=0.1$, the wave associated to the He-component of the mixture appears to be a single component gas-based sound mode for each value of $N_{12}$, since the velocity field $\mathrm{v}_{z}^{1}$ for helium is the same as that obtained for a simple monatomic gas. The results presented in Figures 5-6 can be more deeply evaluated through an analysis of the integral equations given by Eqs. (36) and (37). In the near-free molecular flow regime, the integrals appearing in Eqs. (36) and (37) vanish and the velocity profiles of both components of the mixture are given by the same expression except that the Abramowitz functions depend on $\gamma$, in the case of $\mathrm{v}_{z}^{1}$, and on $\tilde{\gamma}$, in the case of $\mathrm{v}_{z}^{2}$. Therefore, for mixtures like Ne-Ar, whose constituents have comparable molecular mass, the parameters $\gamma$ and $\tilde{\gamma}$ take close values and the macroscopic velocity fields $\mathrm{v}_{z}^{1}$ and $\mathrm{v}_{z}^{2}$ have a similar shape (as 

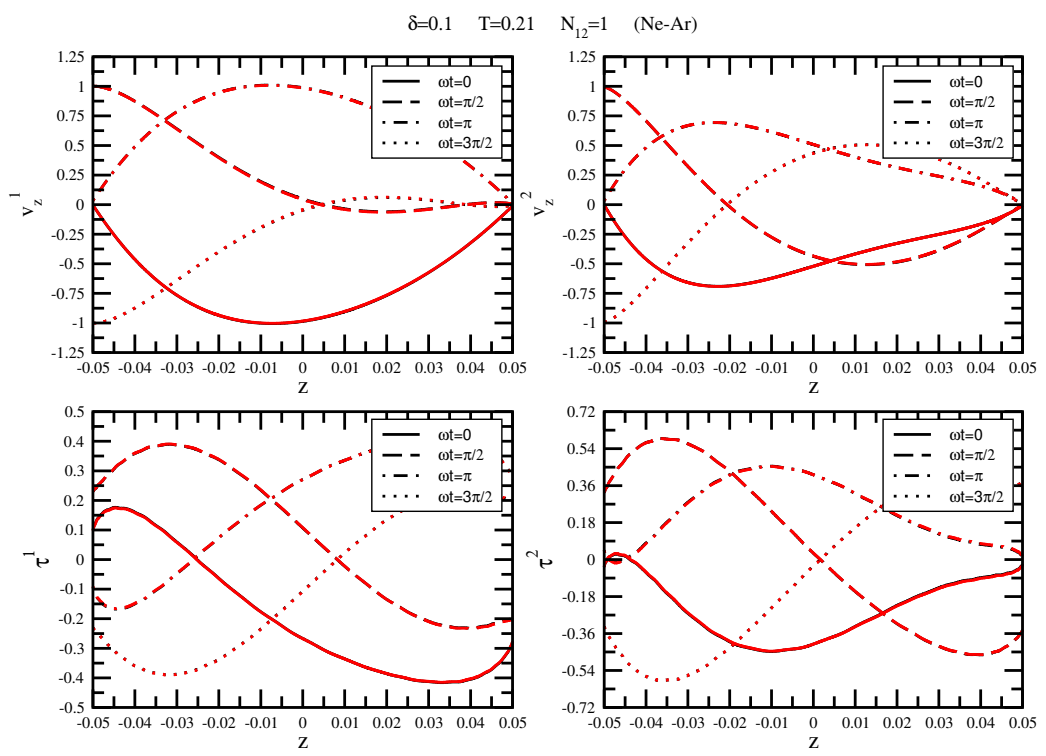

Fig. 5 Variation of the macroscopic velocities and temperatures of the $\mathrm{Ne}-\mathrm{Ar}$ mixture components (with the same molar concentrations), in the $z$-direction across the gap of the channel for $\delta=0.1$ and $T=0.21$. Comparison between the BGK model (black lines) and the McCormack model (red lines). In each panel, the profiles of the macroscopic quantities are shown at different stages during a period of oscillation of the moving wall.

shown in Fig. 5). On the contrary, for disparate-mass gas mixtures like He-Xe, the Abramowitz functions, which depend on $\tilde{\gamma}$, approach zero more quickly as functions of $z$ than those depending on $\gamma$, and the field $v_{z}^{2}$ represents a damped soundlike mode associated to the heaviest component of the mixture (as shown in Fig. 6). However, also in this case, the same remarks valid for $\delta=10$ can be applied: when the period $T$ is very small (of the order of $10^{-2}$ ) both modes associated to the two mixture components are damped, while when $T$ increases, they tend to equilibrate. This result confirms that the equilibration of the modes associated with the two species is not related to the number of collisions between molecules, which, in the near free-molecular flow regime, is small. The same considerations valid for $\delta=10$ and $\delta=0.1$, concerning the characteristics of sound modes excited in the two different mixtures taken into account, hold also for each $\delta$ lying in the transitional region (that is, $0.1<\delta<10$ ).

\section{Concluding Remarks}

In the present paper, we have analyzed the high-frequency sound wave propagation in binary mixtures flowing through microchannels, within the framework of 

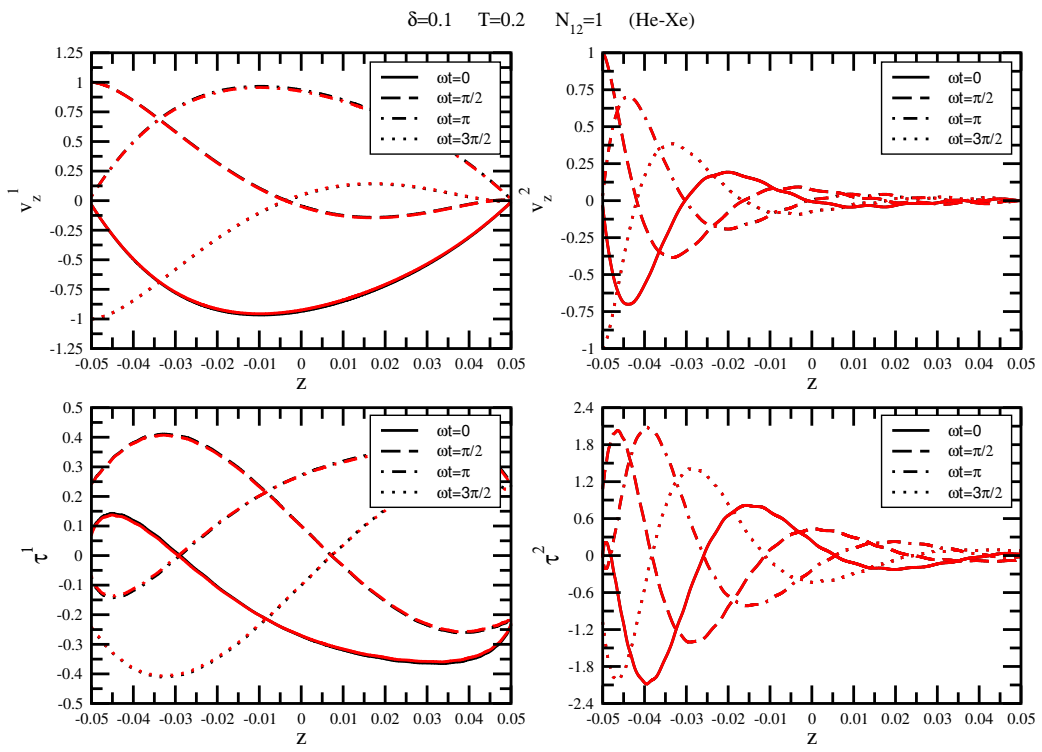

Fig. 6 Variation of the macroscopic velocities and temperatures of the He-Xe mixture components (with the same molar concentrations), in the $z$-direction across the gap of the channel for $\delta=0.1$ and $T=0.2$. Comparison between the BGK model (black lines) and the McCormack model (red lines). In each panel, the profiles of the macroscopic quantities are shown at different stages during a period of oscillation of the moving wall.

kinetic theory of rarefied gases. The results presented refer to mixtures whose constituents have comparable molecular mass (like $\mathrm{Ne}-\mathrm{Ar}$ ) as well as to disparate-mass gas mixtures (composed of very heavy plus very light molecules, like $\mathrm{He}-\mathrm{Xe}$ ). In order to clarify the long standing open question about the precise nature of sound modes excited in binary gas mixtures, we have performed our analysis using the linearized Boltzmann equation based on a simplified kinetic model of BGK-type amenable to a relatively simple semi-analytical manipulation. In this way, through a direct comparison with the integral equations derived from the BGK model for a single-component gas, we were able to infer under what conditions the sound modes associated to the components of the mixture reduce to a single gas-based sound mode. In order to assess the reliability of our findings, since the BGK kinetic model considered in the present paper has been derived in closed-form by assuming Maxwell molecules, we have performed a comparison with the outputs of the McCormack model based on the more realistic assumption of intermolecular rigid-sphere interactions. The results presented in Figures 1-6 show that there is a very good agreement between the outcomes of the BGK and McCormack models. A slight deviation can be observed for the He-Xe mixture in the near-continuum regime when the period of oscillation $T$ increases, but the pictures reveal that the main conclusions drawn by using the BGK model are all confirmed. 
Acknowledgements Silvia Lorenzani is supported by GNFM of INDAM, Italy.

\section{Appendix 1}

The components $k_{i, j}$ of the kernel $\mathrm{K}\left(c_{z}, c_{z}^{\prime}\right)$ required in Eq. (39) read

$$
\begin{aligned}
& k_{1,1}=\frac{2}{3}\left(c_{z}^{2}-\frac{1}{2}\right)\left(c_{z}^{\prime 2}-\frac{1}{2}\right)\left[1-\frac{\left(2 \Gamma^{1} M_{12}\right)}{\left(1+M_{12}\right)}\right] \frac{e^{-c_{z}^{\prime 2}}}{\sqrt{\pi}}+\frac{e^{-c_{z}^{\prime 2}}}{\sqrt{\pi}}+2 c_{z} c_{z}^{\prime}\left(1-\Gamma^{1}\right) \frac{e^{-c_{z}^{\prime 2}}}{\sqrt{\pi}} \\
& k_{1,2}=\frac{2}{3}\left(c_{z}^{2}-\frac{1}{2}\right)\left[1-\frac{\left(2 \Gamma^{1} M_{12}\right)}{\left(1+M_{12}\right)}\right] \frac{e^{-c_{z}^{\prime 2}}}{\sqrt{\pi}} \\
& k_{1,3}=\frac{2}{3}\left(c_{z}^{2}-\frac{1}{2}\right)\left(\frac{c_{z}^{\prime 2}}{M_{12}}-\frac{1}{2}\right)\left[\frac{2 \Gamma^{1} M_{12}}{1+M_{12}}\right] \frac{e^{-c_{z}^{\prime 2} / M_{12}}}{\sqrt{\pi M_{12}}}+2 c_{z} c_{z}^{\prime} \Gamma^{1} \frac{e^{-c_{z}^{\prime 2} / M_{12}}}{\sqrt{\pi M_{12}}} \\
& k_{1,4}=\frac{2}{3}\left(c_{z}^{2}-\frac{1}{2}\right)\left[\frac{2 \Gamma^{1} M_{12}}{1+M_{12}}\right] \frac{e^{-c_{z}^{\prime 2} / M_{12}}}{\sqrt{\pi M_{12}}} \\
& k_{2,1}=\frac{2}{3}\left(c_{z}^{\prime 2}-\frac{1}{2}\right)\left[1-\frac{\left(2 \Gamma^{1} M_{12}\right)}{\left(1+M_{12}\right)}\right] \frac{e^{-c_{z}^{\prime 2}}}{\sqrt{\pi}} \\
& k_{2,2}=\frac{2}{3}\left[1-\frac{\left(2 \Gamma^{1} M_{12}\right)}{\left(1+M_{12}\right)}\right] \frac{e^{-c_{z}^{\prime 2}}}{\sqrt{\pi}} \\
& k_{2,3}=\frac{2}{3}\left(\frac{c_{z}^{\prime 2}}{M_{12}}-\frac{1}{2}\right)\left[\frac{2 \Gamma^{1} M_{12}}{1+M_{12}}\right] \frac{e^{-c_{z}^{\prime 2} / M_{12}}}{\sqrt{\pi M_{12}}} \\
& k_{2,4}=\frac{2}{3}\left[\frac{2 \Gamma^{1} M_{12}}{1+M_{12}}\right] \frac{e^{-c_{z}^{\prime 2} / M_{12}}}{\sqrt{\pi M_{12}}} \\
& k_{3,1}=\frac{2}{3}\left(\frac{c_{z}^{2}}{M_{12}}-\frac{1}{2}\right)\left(c_{z}^{\prime 2}-\frac{1}{2}\right)\left[\frac{2 \Gamma^{2}}{1+M_{12}}\right] \frac{e^{-c_{z}^{\prime 2}}}{\sqrt{\pi}}+2 c_{z} c_{z}^{\prime} \frac{\Gamma^{2}}{M_{12}} \frac{e^{-c_{z}^{\prime 2}}}{\sqrt{\pi}} \\
& k_{3,2}=\frac{2}{3}\left(\frac{c_{z}^{2}}{M_{12}}-\frac{1}{2}\right)\left[\frac{2 \Gamma^{2}}{1+M_{12}}\right] \frac{e^{-c_{z}^{\prime 2}}}{\sqrt{\pi}} \\
& k_{3,3}=\frac{2}{3}\left(\frac{c_{z}^{2}}{M_{12}}-\frac{1}{2}\right)\left(\frac{c_{z}^{\prime 2}}{M_{12}}-\frac{1}{2}\right)\left[1-\frac{\left(2 \Gamma^{2}\right)}{\left(1+M_{12}\right)}\right] \frac{e^{-c_{z}^{\prime 2} / M_{12}}}{\sqrt{\pi M_{12}}}+\frac{e^{-c_{z}^{\prime 2} / M_{12}}}{\sqrt{\pi M_{12}}}
\end{aligned}
$$




$$
\begin{gathered}
+2 c_{z} c_{z}^{\prime} \frac{\left(1-\Gamma^{2}\right)}{M_{12}} \frac{e^{-c_{z}^{\prime 2} / M_{12}}}{\sqrt{\pi M_{12}}} \\
k_{3,4}=\frac{2}{3}\left(\frac{c_{z}^{2}}{M_{12}}-\frac{1}{2}\right)\left[1-\frac{\left(2 \Gamma^{2}\right)}{\left(1+M_{12}\right)}\right] \frac{e^{-c_{z}^{\prime 2} / M_{12}}}{\sqrt{\pi M_{12}}} \\
k_{4,1}=\frac{2}{3}\left(c_{z}^{\prime 2}-\frac{1}{2}\right)\left[\frac{2 \Gamma^{2}}{1+M_{12}}\right] \frac{e^{-c_{z}^{\prime 2}}}{\sqrt{\pi}} \\
k_{4,2}=\frac{2}{3}\left[\frac{2 \Gamma^{2}}{1+M_{12}}\right] \frac{e^{-c_{z}^{\prime 2}}}{\sqrt{\pi}} \\
k_{4,3}=\frac{2}{3}\left(\frac{c_{z}^{\prime 2}}{M_{12}}-\frac{1}{2}\right)\left[1-\frac{\left(2 \Gamma^{2}\right)}{\left(1+M_{12}\right)}\right] \frac{e^{-c_{z}^{\prime 2} / M_{12}}}{\sqrt{\pi M_{12}}} \\
k_{4,4}=\frac{2}{3}\left[1-\frac{\left(2 \Gamma^{2}\right)}{\left(1+M_{12}\right)}\right] \frac{e^{-c_{z}^{\prime 2} / M_{12}}}{\sqrt{\pi M_{12}}}
\end{gathered}
$$

\section{Appendix 2}

In 1973, F. J. McCormack proposed in [20] a simple method of construction of linearized kinetic models of the Boltzmann equation for gas mixtures based upon the equivalence of moments of the $N$ th-order modeled collision operator and the moments of the full collision operator calculated with the $N$ th-order approximation to the distribution function. This method has been then used to construct a third-order model (which is the lowest order that gives a correct hydrodynamic description) of the linearized cross-collision operator for a general multicomponent monatomic gaseous mixture, known in the literature as 'McCormack model'. For the flow problem under investigation, the McCormack linearized collision operator reads

$$
\begin{gathered}
L h^{1}{ }_{M C}=\gamma_{1}\left\{\rho^{1}+2\left(1-\eta_{1,2}^{1}\right) \mathbf{c} \cdot \mathbf{v}^{1}-2 \eta_{1,2}^{2} \mathbf{c} \cdot \mathbf{q}^{1}+\left[1-\frac{2 \eta_{1,2}^{1} M_{12}}{\left(1+M_{12}\right)}\right]\left(|\mathbf{c}|^{2}-\frac{3}{2}\right) \tau^{1}\right. \\
+2\left(1+\eta_{1,1}^{4}-\eta_{1,1}^{3}-\eta_{1,2}^{3}\right) c_{i} c_{j} \tilde{P}_{i j}^{1}+\frac{8}{5}\left(1+\eta_{1,1}^{6}-\eta_{1,1}^{5}-\eta_{1,2}^{5}\right)\left(|\mathbf{c}|^{2}-\frac{5}{2}\right) \mathbf{c} \cdot \mathbf{q}^{1} \\
-\eta_{1,2}^{2}\left(|\mathbf{c}|^{2}-\frac{5}{2}\right) \mathbf{c} \cdot \mathbf{v}^{1}+2 \eta_{1,2}^{1} \mathbf{c} \cdot \mathbf{v}^{2}+2 M_{12} \eta_{1,2}^{2} \mathbf{c} \cdot \mathbf{q}^{2}+\eta_{1,2}^{2}\left(|\mathbf{c}|^{2}-\frac{5}{2}\right) \mathbf{c} \cdot \mathbf{v}^{2} \\
\left.+\frac{2 \eta_{1,2}^{1} M_{12}}{\left(1+M_{12}\right)}\left(|\mathbf{c}|^{2}-\frac{3}{2}\right) \tau^{2}+\frac{2 \eta_{1,2}^{4}}{M_{12}} c_{i} c_{j} \tilde{P}_{i j}^{2}+\frac{8 \eta_{1,2}^{6}}{5 \sqrt{M_{12}}}\left(|\mathbf{c}|^{2}-\frac{5}{2}\right) \mathbf{c} \cdot \mathbf{q}^{2}-h^{1}\right\}
\end{gathered}
$$




$$
\begin{aligned}
& L h^{2}{ }_{M C}=\gamma_{2}\left\{\rho^{2}+\frac{2}{M_{12}}\left(1-\eta_{2,1}^{1}\right) \mathbf{c} \cdot \mathbf{v}^{2}-\frac{2 \eta_{2,1}^{2}}{M_{12}} \mathbf{c} \cdot \mathbf{q}^{2}+\left[1-\frac{2 \eta_{2,1}^{1}}{\left(1+M_{12}\right)}\right]\left(\frac{|\mathbf{c}|^{2}}{M_{12}}-\frac{3}{2}\right) \tau^{2}\right. \\
& +\frac{2}{M_{12}^{2}}\left(1+\eta_{2,2}^{4}-\eta_{2,2}^{3}-\eta_{2,1}^{3}\right) c_{i} c_{j} \tilde{P}_{i j}^{2}+\frac{8}{5 M_{12}}\left(1+\eta_{2,2}^{6}-\eta_{2,2}^{5}-\eta_{2,1}^{5}\right)\left(\frac{|\mathbf{c}|^{2}}{M_{12}}-\frac{5}{2}\right) \mathbf{c} \cdot \mathbf{q}^{2} \\
& \quad-\frac{\eta_{2,1}^{2}}{M_{12}}\left(\frac{|\mathbf{c}|^{2}}{M_{12}}-\frac{5}{2}\right) \mathbf{c} \cdot \mathbf{v}^{2}+\frac{2 \eta_{2,1}^{1}}{M_{12}} \mathbf{c} \cdot \mathbf{v}^{1}+\frac{2 \eta_{2,1}^{2}}{M_{12}^{2}} \mathbf{c} \cdot \mathbf{q}^{1}+\frac{\eta_{2,1}^{2}}{M_{12}}\left(\frac{|\mathbf{c}|^{2}}{M_{12}}-\frac{5}{2}\right) \mathbf{c} \cdot \mathbf{v}^{1} \\
& \left.+\frac{2 \eta_{2,1}^{1}}{\left(1+M_{12}\right)}\left(\frac{|\mathbf{c}|^{2}}{M_{12}}-\frac{3}{2}\right) \tau^{1}+\frac{2 \eta_{2,1}^{4}}{M_{12}} c_{i} c_{j} \tilde{P}_{i j}^{1}+\frac{8 \eta_{2,1}^{6}}{5 \sqrt{M_{12}}}\left(\frac{|\mathbf{c}|^{2}}{M_{12}}-\frac{5}{2}\right) \mathbf{c} \cdot \mathbf{q}^{1}-h^{2}\right\}
\end{aligned}
$$

where

$$
\eta_{s, r}^{k}=\frac{v_{s, r}^{k}}{\gamma_{s}} \quad(s, r=1,2 \quad k=1, \ldots, 6)
$$

and the collision frequencies $\gamma_{s}(s=1,2)$ are expressed as

$$
\begin{aligned}
& \gamma_{1}=\left(\psi_{1} \psi_{2}-v_{1,2}^{4} v_{2,1}^{4}\right)\left(\psi_{2}+v_{1,2}^{4}\right)^{-1} \\
& \gamma_{2}=\left(\psi_{1} \psi_{2}-v_{1,2}^{4} v_{2,1}^{4}\right)\left(\psi_{1}+v_{2,1}^{4}\right)^{-1}
\end{aligned}
$$

with

$$
\begin{aligned}
& \psi_{1}=v_{1,1}^{3}+v_{1,2}^{3}-v_{1,1}^{4} \\
& \psi_{2}=v_{2,2}^{3}+v_{2,1}^{3}-v_{2,2}^{4}
\end{aligned}
$$

In addition

$$
\begin{gathered}
v_{s, r}^{1}=\frac{16}{3} \frac{\mu^{s r}}{m^{s}} n^{r} \Omega_{s, r}^{11}, \\
v_{s, r}^{2}=\frac{64}{15}\left(\frac{\mu^{s r}}{m^{s}}\right)^{2} n^{r}\left(\Omega_{s, r}^{12}-\frac{5}{2} \Omega_{s, r}^{11}\right), \\
v_{s, r}^{3}=\frac{16}{5}\left(\frac{\mu^{s r}}{m^{s}}\right)^{2} \frac{m^{s}}{m^{r}} n^{r}\left(\frac{10}{3} \Omega_{s, r}^{11}+\frac{m^{r}}{m^{s}} \Omega_{s, r}^{22}\right), \\
v_{s, r}^{4}=\frac{16}{5}\left(\frac{\mu^{s r}}{m^{s}}\right)^{2} \frac{m^{s}}{m^{r}} n^{r}\left(\frac{10}{3} \Omega_{s, r}^{11}-\Omega_{s, r}^{22}\right), \\
v_{s, r}^{5}=\frac{64}{15}\left(\frac{\mu^{s r}}{m^{s}}\right)^{3} \frac{m^{s}}{m^{r}} n^{r} \Gamma_{s, r}^{5}, \\
v_{s, r}^{6}=\frac{64}{15}\left(\frac{\mu^{s r}}{m^{s}}\right)^{3}\left(\frac{m^{s}}{m^{r}}\right)^{3 / 2} n^{r} \Gamma_{s, r}^{6}
\end{gathered}
$$

with

$$
\Gamma_{s, r}^{5}=\Omega_{s, r}^{22}+\left(\frac{15 m^{s}}{4 m^{r}}+\frac{25 m^{r}}{8 m^{s}}\right) \Omega_{s, r}^{11}-\left(\frac{m^{r}}{2 m^{s}}\right)\left(5 \Omega_{s, r}^{12}-\Omega_{s, r}^{13}\right),
$$




$$
\Gamma_{s, r}^{6}=-\Omega_{s, r}^{22}+\frac{55}{8} \Omega_{s, r}^{11}-\frac{5}{2} \Omega_{s, r}^{12}+\frac{1}{2} \Omega_{s, r}^{13} .
$$

The $\Omega_{s, r}^{i j}$ are the Chapman-Cowling integrals [8], which for the rigid sphere interaction read

$$
\Omega_{s, r}^{i j}=\frac{(j+1) !}{8}\left[1-\frac{1+(-1)^{i}}{2(i+1)}\right]\left(\frac{\pi k T}{2 \mu^{s r}}\right)^{1 / 2}\left(d_{s}+d_{r}\right)^{2}
$$

where $d_{s}(s=1,2)$ are the molecular diameters of every species $s$. Furthermore, the dimensionless macroscopic perturbed density $\left(\rho^{s}\right)$, velocity $\left(\mathbf{v}^{s}\right)$, temperature $\left(\tau^{s}\right)$, stress tensor $\left(\tilde{P}_{i j}^{s}\right)$ and heat flux $\left(\mathbf{q}^{s}\right)$, appearing in the McCormack model, are defined as follows

$$
\begin{gathered}
\rho^{1}=\frac{1}{\pi^{3 / 2}} \int_{\mathbf{R}^{3}} h^{1} \mathrm{e}^{-|\mathbf{c}|^{2}} d \mathbf{c}, \quad \rho^{2}=\frac{1}{\left(\pi M_{12}\right)^{3 / 2}} \int_{\mathbf{R}^{3}} h^{2} \mathrm{e}^{-\frac{|\mathbf{c}|^{2}}{M_{12}}} d \mathbf{c} \\
\mathbf{v}^{1}=\frac{1}{\pi^{3 / 2}} \int_{\mathbf{R}^{3}} \mathbf{c} h^{1} \mathrm{e}^{-|\mathbf{c}|^{2}} d \mathbf{c}, \quad \mathbf{v}^{2}=\frac{1}{\left(\pi M_{12}\right)^{3 / 2}} \int_{\mathbf{R}^{3}} \mathbf{c} h^{2} \mathrm{e}^{-\frac{|\mathbf{c}|^{2}}{M_{12}}} d \mathbf{c} \\
\tau^{1}=\frac{1}{\pi^{3 / 2}} \int_{\mathbf{R}^{3}}\left(\frac{2}{3}|\mathbf{c}|^{2}-1\right) h^{1} \mathrm{e}^{-|\mathbf{c}|^{2}} d \mathbf{c} \\
\tau^{2}=\frac{1}{\left(\pi M_{12}\right)^{3 / 2}} \int_{\mathbf{R}^{3}}\left(\frac{2}{3 M_{12}}|\mathbf{c}|^{2}-1\right) h^{2} \mathrm{e}^{-\frac{|\mathbf{c}|^{2}}{M_{12}}} d \mathbf{c} \\
\tilde{P}_{i j}^{1}=\frac{1}{\pi^{3 / 2}} \int_{\mathbf{R}^{3}}\left(c_{i} c_{j}-\frac{1}{3}|\mathbf{c}|^{2} \delta_{i j}\right) h^{1} \mathrm{e}^{-|\mathbf{c}|^{2}} d \mathbf{c} \\
\tilde{P}_{i j}^{2}=\frac{1}{\left(\pi M_{12}\right)^{3 / 2}} \int_{\mathbf{R}^{3}}\left(c_{i} c_{j}-\frac{1}{3}|\mathbf{c}|^{2} \delta_{i j}\right) h^{2} \mathrm{e}^{-\frac{\left.\mathbf{c}\right|^{2}}{M_{12}}} d \mathbf{c} \\
\mathbf{q}^{1}=\frac{1}{\pi^{3 / 2}} \int_{\mathbf{R}^{3}} \frac{1}{2} \mathbf{c}\left(|\mathbf{c}|^{2}-\frac{5}{2}\right) h^{1} \mathrm{e}^{-|\mathbf{c}|^{2}} d \mathbf{c} \\
\mathbf{q}^{2}=\frac{1}{\left(\pi M_{12}\right)^{3 / 2}} \int_{\mathbf{R}^{3}} \frac{1}{2} \mathbf{c}\left(\frac{|\mathbf{c}|^{2}}{M_{12}}-\frac{5}{2}\right) h^{2} \mathrm{e}^{-\frac{|\mathbf{c}|^{2}}{M_{12}}} d \mathbf{c}
\end{gathered}
$$

\section{References}

1. Andries, P., Aoki, K., Perthame, B.: A consistent BGK-type model for gas mixtures. J. Stat. Phys. 106, 993-1018 (2002)

2. Bisi, M., Lorenzani, S.: High-frequency sound wave propagation in binary gas mixtures flowing through microchannels. Phys. Fluids 28, 052003 (2016)

3. Bowler, J.R., Johnson, E.A.: Anomalous dispersion in disparate-mass gas mixtures. Phys. Rev. Lett. 54, 329-332 (1985)

4. Cercignani, C.: Mathematical methods in kinetic theory. Plenum, New York (1969)

5. Cercignani, C., Daneri, A.: Flow of a rarefied gas between two parallel plates. J. Appl. Phys. 34, 3509-3513 (1963) 
6. Cercignani, C., Sernagiotto, F.: The method of elementary solutions for time dependent problems in linearized kinetic theory. Ann. Phys. 30, 154-167 (1964)

7. Cercignani, C., Lampis, M., Lorenzani, S.: Plane Poiseuille-Couette problem in microelectro-mechanical systems applications with gas-rarefaction effects. Phys. Fluids 18, 087102 (2006)

8. Chapman, S., Cowling, T.G.: The Mathematical Theory of Non-Uniform Gases. Cambridge University Press, New York (1970)

9. Desvillettes, L., Lorenzani, S.: Sound wave resonances in micro-electro-mechanical systems devices vibrating at high frequencies according to the kinetic theory of gases. Phys. Fluids 24, 092001 (2012)

10. Garcia, R.D.M., Siewert, C.E.: The linearized Boltzmann equation: Sound-wave propagation in a rarefied gas. ZAMP 57, 94-122 (2006)

11. Gosse, L.: Computing qualitatively correct approximations of balance laws. Springer, Milan (2013)

12. Grad, H.: Theory of rarefied gases. In: Devienne, F.M. (ed.) Rarefied Gas Dynamics, pp. 100-138. Pergamon, New York (1960)

13. Groppi, M., Spiga, G.: A Bhatnagar-Gross-Krook-type approach for chemically reacting gas mixtures. Phys. Fluids 16, 4273-4284 (2004)

14. Huck, R.J., Johnson, E.A.: Possibility of double sound propagation in disparate-mass gas mixtures. Phys. Rev. Lett. 44, 142-145 (1980)

15. Kalempa, D., Sharipov, F.: Sound propagation through a rarefied gas confined between source and receptor at arbitrary Knudsen number and sound frequency. Phys. Fluids 21, 103601 (2009)

16. Landau, L.D., Lifshitz, E.M.: Fluid Mechanics. Pergamon, New York (1989)

17. Lighthill, J.: Waves in Fluids. Cambridge University Press, New York (1978)

18. Lorenzani, S., Gibelli, L., Frezzotti, A., Frangi, A., Cercignani, C.: Kinetic approach to gas flows in microchannels. Nanoscale Microscale Thermophys. Eng. 11, 211-226 (2007)

19. Loyalka, S.K., Cheng, T.C.: Sound-wave propagation in a rarefied-gas. Phys. Fluids 22, 830 836 (1979)

20. McCormack, F.J.: Construction of linearized kinetic models for gaseous mixtures and molecular gases. Phys. Fluids 16, 2095-2105 (1973)

21. Naris, S., Valougeorgis, D., Kalempa, D., Sharipov, F.: Gaseous mixture flow between two parallel plates in the whole range of the gas rarefaction. Physica A 336, 294-318 (2004)

22. Naris, S., Valougeorgis, D., Kalempa, D., Sharipov, F.: Flow of gaseous mixtures through rectangular microchannels driven by pressure, temperature, and concentration gradients. Phys. Fluids 17, 100607 (2005)

23. Siewert, C.E., Valougeorgis, D.: The McCormack model: channel flow of a binary gas mixture driven by temperature, pressure and density gradients. European J. Mechanics B/Fluids 23, 645-664 (2004)

24. Siewert, C.E., Valougeorgis, D.: Concise and accurate solutions to half-space binary-gas flow problems defined by the McCormack model and specular-diffuse wall conditions. European J. Mechanics B/Fluids 23, 709-726 (2004)

25. Sirovich, L., Thurber, J.K.: Propagation of forced sound waves in rarefied gasdynamics. J. Acoust. Soc. Am. 37, 329-339 (1965) 